
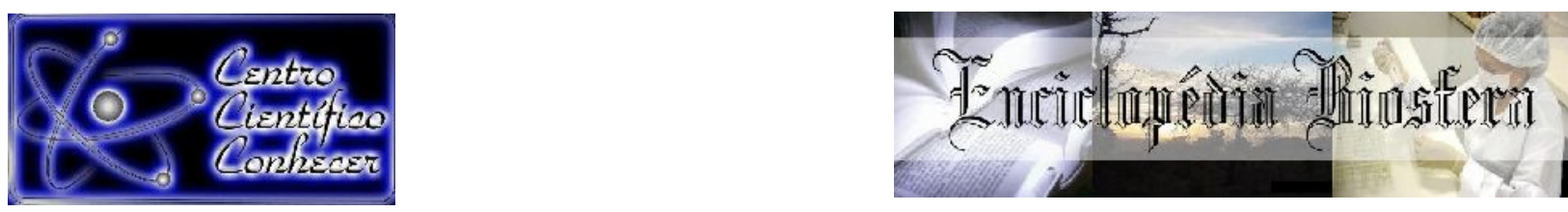

\title{
PERFIL DE ANSIEDADE EM SERVIDORES DE UMA INSTITUIÇÃO DE ENSINO SUPERIOR DO NOROESTE DE MINAS GERAIS
}

Karielly Amaral Andrade1; Amanda Melo Sant'Anna Araújo²; Eric Francelino Andrade $^{3}$; Débora Ribeiro Orlando ${ }^{4}$

1 Graduanda em Medicina Veterinária, Universidade Federal dos Vales do Jequitinhonha e Mucuri (UFVJM) - Instituto de Ciências Agrárias Unaí, MG - Brasil. 2 Prof. Mestre, Universidade Federal dos Vales do Jequitinhonha e Mucuri (UFVJM) - Instituto de Ciências Agrárias, Unaí, MG - Brasil.

3 Prof. Doutor, Universidade Federal dos Vales do Jequitinhonha e Mucuri (UFVJM)

- Instituto de Ciências Agrárias, Unaí, MG - Brasil.

4 Profa. Doutora, Universidade Federal dos Vales do Jequitinhonha e Mucuri (UFVJM) - Instituto de Ciências Agrárias/Campus Unaí-MG Avenida Universitária, 1000, Universitários, Unaí, MG - Brasil (deboraribeiro.orlando@gmail.com)

\section{Recebido em: 15/05/2020 - Aprovado em: 15/06/2020 - Publicado em: 30/06/2020} DOI: 10.18677/EnciBio_2020B38

\begin{abstract}
RESUMO
A ansiedade é um transtorno que pode promover prejuízos na qualidade de vida e de trabalho. Considerando, que servidores da área de educação superior estão susceptíveis a agentes estressores que provocam ansiedade, objetivou-se com o presente estudo, avaliar o perfil de ansiedade em servidores públicos de uma Universidade do noroeste do estado de Minas Gerais. Participaram do estudo 61 servidores (32 docentes e 29 técnicos) com idade média de 34,8 $\pm 5,4$ anos. Os voluntários responderam ao Inventário de Ansiedade Traço-Estado (IDATE), que é um instrumento que permite avaliar e classificar o nível de ansiedade. Os dados obtidos foram submetidos à estatística descritiva e à análise de variância (ANOVA) e teste do qui-quadrado e as médias comparadas pelo teste de te student $(p<0,05)$. A maior parte dos servidores foi classificada com baixo nível de ansiedade, tanto traço quanto estado $(p<0,05)$. Os indivíduos do sexo feminino apresentaram maiores níveis de ansiedade-estado em comparação aos homens e os servidores com mais de 12 meses de serviço apresentaram maiores escores de ansiedade $(p<$ 0,05). Não houve diferenças significativas nos escores de ansiedade quando se comparou docente e técnico. Conclui-se que, dentre os servidores avaliados, nos níveis de ansiedade foram maiores em mulheres e naqueles com maior tempo de serviço público.
\end{abstract}

PALAVRAS-CHAVE: Ansiedade, professores, servidores públicos. 


\title{
PROFILE OF ANXIETY IN WORKERS OF A HIGHER EDUCATION INSTITUTION IN THE NORTHWEST OF MINAS GERAIS
}

\begin{abstract}
Anxiety is a mood disorder that can impair quality of life and work capacity. Considering that civil servants of higher education area are susceptible to some stressors that cause anxiety, we aimed to evaluate the profile of anxiety in workers of a University situated in the northwest of the state of Minas Gerais. Sixty-one civil servants (32 teachers and 29 technicians) with $34.8 \pm 5.4$ years of age participated of the study. The volunteers responded to the State-Trait Anxiety Inventory (STAI), which is an instrument that allows assessing and classifying the level of anxiety. The data obtained were submitted to descriptive statistics, analysis of variance (ANOVA) and the chi-square test and the means was compared by the student's t test ( $p$ $<0.05)$. The most of workers were classified with low levels of anxiety, both trait and state $(p<0.05)$. Female individuals had higher levels of anxiety-state compared to men and employees with more than 12 months of service had higher anxiety scores $(p<0.05)$ There were no significant differences in anxiety scores when comparing teachers and technical. We concluded that, among the workers evaluated, the levels of anxiety were higher in women and in those with most time in public service.
\end{abstract}

KEYWORDS: Anxiety. Pet. University Students.

\section{INTRODUÇÃO}

A ansiedade é uma reação adaptativa que ocorre diante de alguma ameaça, seja ela real ou não (ROBERTS; DUONG, 2017). Quando esta reação ocorre de forma crônica ou em elevada intensidade, podem ocorrer efeitos deletérios para o organismo (REUMAN; ABRAMOWITZ, 2018). Dentre estes efeitos, pode-se destacar a piora cognitiva e na memória, bem como diminuição na capacidade de trabalho (MUSCHALLA, 2016; SARI et al., 2017). Fisiologicamente, a ansiedade desencadeia uma resposta de luta ou fuga que promove a ativação do eixo hipotálamo-hipófiseadrenal, culminando na secreção do hormônio cortisol (FIKSDAL et al., 2019). Este hormônio é importante para o controle de substratos energéticos circulantes e da homeostasia (RAMAMOORTHY; CIDLOWSKI, 2016). Todavia, quando a secreção é frequente ou aumentada, podem ocorrer distúrbios metabólicos como dislipidemias e diabetes mellitus, além da supressão da resposta imunológica, fazendo com que o indivíduo fique mais propenso a infecções (RAMAMOORTHY; CIDLOWSKI, 2016).

No setor laboral, a ansiedade pode acarretar afastamentos por saúde e baixa na produtividade (MCCARTHY et al., 2016). Desta forma, transtornos de ansiedade acarretam uma alta demanda de assistência profissional, o que demonstra a importância para a saúde pública (KULSOOM; AFSAR, 2015). Adicionalmente, a ansiedade é um dos quadros psiquiátricos mais comuns que atinge todos os extratos da sociedade (REMES et al., 2016).

Dentre as profissões consideradas mais estressantes, e que por sua vez podem gerar maiores níveis de ansiedade, pode-se destacar a docência (JOHNSON et al., 2005). Professores, geralmente, têm altos desafios físicos e mentais devido à elevada carga de trabalho, planejamento de aulas, organização de atividades, orientação de alunos, dentre outros (DESOUKY; ALLAM, 2017). Adicionalmente, profissionais envolvidos com o setor educacional são propensos em desenvolver ansiedade (MUSCHALLA; LINDEN, 2013) e isso pode ser potencializado quando se trata de servidores públicos (LOPES; DA SILVA, 2018). 
Dentre os aspectos que predispõe a ansiedade no trabalho, em funcionários públicos, destacam-se a pressão para atingir objetivos, normas e regulações institucionais e cobrança por produção (BASTOS et al., 2018). Além disso, esta população de trabalhadores pode se sentir injustiçada a ansiosa por sua imagem pública estar, muitas vezes, associada à apatia e ineficiência (LEÃO et al., 2015; BASTOS et al., 2018).

Desta forma, considerando que servidores públicos da área educacional estão susceptíveis a fatores que promovem ansiedade, objetivou-se com o presente estudo, avaliar o perfil de ansiedade em professores e técnicos de uma Universidade localizada no noroeste de Minas Gerais.

\section{Participantes}

\section{MATERIAIS E MÉTODOS}

Participaram do estudo 61 servidores (32 docentes e 29 técnicos) do Instituto de Ciências Agrárias da Universidade Federal dos Vales do Jequitinhonha e Mucuri. A idade dos participantes variou de 24 a 47 anos, sendo a média de 34,8 \pm 5,4 anos. Entre os docentes, 22 eram homens e 10 mulheres, enquanto entre os técnicos havia 12 homens e 17 mulheres. A coleta de dados foi realizada em novembro de 2018. A amostra obtida para o presente estudo compreendeu todos os servidores que estavam trabalhando durante o período de coleta dos dados. Não entraram no estudo servidores que estavam em período de férias ou afastados do serviço por qualquer motivo. Os participantes assinaram um Termo de Consentimento Livre e Esclarecido (TCLE) que continha informações sobre os objetivos do estudo bem como a garantia de confidencialidade dos dados informados. O conteúdo do TCLE também foi explicado verbalmente antes da aplicação do questionário, que foi aplicado individualmente.

\section{Instrumento}

Para avaliação da ansiedade, foi utilizado o Inventário de Ansiedade TraçoEstado (IDATE), desenvolvido por Spielberger et al. (1970) e validado para a população brasileira por Biaggio et al. (1977). Este instrumento permite avaliar a ansiedade entre suas vertentes, ansiedade-estado (AE) e ansiedade-traço (AT) e apresenta 40 questões. Vinte questões são relacionadas à AT enquanto outras 20 relacionadas à $\mathrm{AE}$. Cada questão permite as seguintes respostas em escala de Likert: "muitíssimo", "bastante", "um pouco", e "absolutamente não". O nível de ansiedade é determinado pelo somatório dos valores atribuídos às respostas das questões tanto para AE quanto para AT. Assim, após a conversão das respostas em valores numéricos, o valor mais alto do somatório indica maiores níveis de ansiedade, enquanto valores mais baixos correspondem à menores níveis deste transtorno (LORICCHIO; LEITE, 2012).

Os valores obtidos em cada escala do IDATE apresentam variação entre 20 e 80 pontos, permitindo a classificação de níveis de ansiedade conforme realizado em estudo prévio (GERRETH et al., 2019). Assim, valores entre 20 e 40 foram classificados como baixo nível de ansiedade, entre 41 e 60 como ansiedade moderada e entre 61 e 80 como alto nível de ansiedade (GERRETH et al., 2019).

\section{Análises Estatísticas}

Os dados após classificação, foram submetidos à estatística descritiva e análise de variância (ANOVA) e as médias foram comparadas pelo teste de $\mathrm{t}$ de student $(p<0,05)$. Para comparar a frequência de indivíduos situados em cada nível 
de ansiedade foi utilizado o teste do qui-quadrado. As análises foram realizadas utilizando o pacote do software BIOESTAT 3.0 (AYRES et al., 2003).

\section{RESULTADOS E DISCUSSÃO}

Observou-se que, na amostra geral, os valores médios de ansiedade-estado foram 38,68 $\pm 7,39$, enquanto para ansiedade-traço estes valores foram 33,31 \pm 5,99 . Na classificação geral dos níveis de ansiedade, a maior parte dos servidores foi classificada com baixo nível deste transtorno, tanto para o componente traço quanto para o estado ( $p>0,05-$ Tabela 1). Apenas um servidor apresentou nível alto de ansiedade-estado, enquanto nenhum dos servidores foi classificado com alto nível de ansiedade-traço.

TABELA 1 - Classificação geral quanto ao nível em servidores do Instituto de Ciências Agrárias da Universidade Federal dos Vales do Jequitinhonha e Mucuri (ICA-UFVJM).

\begin{tabular}{cccc}
\hline Classificação do nível de ansiedade & Baixo (n/\%) & Moderado (n/\%) & Alto (n/\%) \\
\hline Ansiedade-Estado & $43(70,49 \%)$ & $17(27,87 \%)$ & $1(1,64 \%)$ \\
Ansiedade-Traço & ${ }^{*} 51(83,61 \%)$ & $10(16,39 \%)$ & $0(0 \%)$ \\
\hline
\end{tabular}

* Maior prevalência de indivíduos localizados no nível de ansiedade $(p<0,05)$.

A ansiedade-estado é relacionada à uma situação momentânea de ansiedade apresentada pelo indivíduo, enquanto a ansiedade-traço é uma característica, relativamente permanente, relacionada à personalidade (LEAL et al., 2017). Neste sentido, a vertente traço da ansiedade é mais estável e mais difícil de ser modificada, enquanto a ansiedade-estado é mais passível de alteração frente à diferentes situações (LEAL et al., 2017). Os resultados observados no presente estudo com relação ao nível de ansiedade dos servidores estão de acordo com o observado por Desouky e Allam (2017) que observaram baixos níveis de ansiedade em professores do Egito utilizando também o IDATE para avaliar este parâmetro.

Considerando os níveis de ansiedade com relação ao gênero, observou-se que na ansiedade-traço, houve maior prevalência, tanto de homens quanto mulheres, classificados com baixo nível de ansiedade (Tabela 2). Na ansiedadeestado observou-se prevalência de indivíduos do sexo masculino classificados com baixo nível de ansiedade, havendo uma frequência maior de mulheres classificadas com ansiedade moderada ( $p<0,05-$ Tabela 2 ).

TABELA 2 - Classificação quanto ao nível de ansiedade em servidores do Instituto de Ciências Agrárias da Universidade Federal dos Vales do Jequitinhonha e Mucuri (ICA-UFVJM - Campus Unaí).

\begin{tabular}{|c|c|c|c|c|}
\hline $\begin{array}{l}\text { Classificação do nível } \\
\text { de ansiedade }\end{array}$ & Gênero & Baixo n (\%) & Moderado (n/\%) & Alto (n/\%) \\
\hline \multirow{2}{*}{ Ansiedade-Estado } & Masculino & $27(79,41 \%)$ & $6(17,64 \%)$ & $1(2,95 \%)$ \\
\hline & Feminino & $16(59,25 \%)$ & ${ }^{*} 11(40,75 \%)$ & $0(0 \%)$ \\
\hline \multirow{2}{*}{ Ansiedade-Traço } & Masculino & $29(85,29 \%)$ & $5(14,71 \%)$ & $0(0 \%)$ \\
\hline & Feminino & *22 (81,48\%) & 5 (18,52\%) & $0(0 \%)$ \\
\hline
\end{tabular}


Os resultados para o nível de ansiedade-traço observados na amostra geral, quando se realizou a estratificação por gênero, estão em consonância com os resultados sem a estratificação, e de acordo com o estudo citado anteriormente (DESOUKY; ALLAM, 2017). A relação entre gênero e predisposição à ansiedade é descrita na literatura, sendo relatada, na maioria das vezes, maior prevalência de mulheres em níveis mais elevados de ansiedade em comparação a seus congêneres (SKAPINAKIS et al., 2013; CARVALHO et al., 2015; UMUZDAŞ et al., 2019).

No presente estudo, não foram observadas diferenças significativas nos escores de ansiedade (estado e traço) na comparação entre função (docente ou técnico). Já com relação ao tempo de serviço prestado na instituição, observou-se que os servidores com mais de 12 meses de serviço público apresentaram maiores escores de ansiedade-estado quando comparados àqueles com menor tempo $(p<$ 0,05 - Tabela 3). Não houve diferença no escore de ansiedade-traço na comparação entre o tempo de serviço (Tabela 3 ).

TABELA 3 - Características do perfil de ansiedade em servidores do Instituto de Ciências Agrárias da Universidade Federal dos Vales do Jequitinhonha e Mucuri (ICA-UFVJM - Campus Unaí).

\begin{tabular}{|c|c|c|c|c|c|c|}
\hline & Escores I & lédios de ansiedade er & serv & Jores $(\mathrm{N}$ & =61) & \\
\hline \multirow{3}{*}{$\begin{array}{l}\text { Ansiedade- } \\
\text { Estado }\end{array}$} & \multirow{5}{*}{ Função } & Grupo & $\mathbf{n}$ & Média & DP & $\mathbf{p}$ \\
\hline & & Docente & 32 & 36.72 & 8.51 & \multirow{2}{*}{0.10} \\
\hline & & Técnico & 29 & 40.86 & 6.10 & \\
\hline \multirow{2}{*}{$\begin{array}{c}\text { Ansiedade- } \\
\text { Traço }\end{array}$} & & Docente & 32 & 33.43 & 6.14 & \multirow{2}{*}{0.89} \\
\hline & & Técnico & 29 & 33.17 & 5.81 & \\
\hline \multirow{2}{*}{$\begin{array}{l}\text { Ansiedade- } \\
\text { Estado }\end{array}$} & & Até 12 meses & 22 & 34.90 & 5.81 & \multirow{2}{*}{0.02} \\
\hline & Tempo de servico & Superior a 12 meses & 39 & 40.82 & 8.29 & \\
\hline \multirow{2}{*}{$\begin{array}{c}\text { Ansiedade- } \\
\text { Traço }\end{array}$} & & Até 12 meses & 22 & 34.31 & 4.37 & \multirow{2}{*}{0.54} \\
\hline & & Superior a 12 meses & 39 & 32.74 & 6.79 & \\
\hline
\end{tabular}

A ansiedade é considerada uma das principais condições de transtorno de humor, responsável pelo afastamento de servidores públicos (SILVA et al., 2012; OLIVEIRA et al., 2015; TREVISAN et al., 2019). No Brasil, é considerado que, mesmo havendo uma suposta estabilidade no trabalho e menor risco de demissão de servidores públicos, estes trabalhadores podem ser afetados pela precarização e degradação de suas condições de trabalho, acúmulo de funções e instabilidade em decorrência de mudanças políticas (NUNES; LINS, 2009; OLIVEIRA et al., 2015). Tais fatores são potenciais agentes estressores que podem levar a quadros de ansiedade nesta população e, potencialmente, se agravar com o tempo de serviço, como observado nos resultados do presente estudo onde foram observados maiores escores de ansiedade nos servidores com mais de 12 meses de serviço público.

\section{CONCLUSÃO}

Conclui-se que os servidores públicos da instituição de ensino superior do sexo feminino e com maior tempo de serviço apresentam maiores níveis de ansiedade. Tais resultados são importantes para se estabelecer abordagens direcionadas para o controle de ansiedade desta população. 


\section{AGRADECIMENTOS}

À Fundação de Amparo à Pesquisa de Minas Gerais (FAPEMIG) pela concessão da bolsa de iniciação científica e à Universidade Federal dos Vales do Jequitinhonha e Mucuri (UFVJM).

\section{REFERÊNCIAS}

AYRES, M.; AYRES JR, M.; AYRES, D.L.; SANTOS, A. S. BioEstat 3.0: aplicação estatísticas nas áreas das ciências biológicas e médicas. Belém:Sociedade Civil Mamirauá; 2003.

BASTOS, M. L. A.; DA SILVA JUNIOR, G. B.; DOMINGOS, E. T. C.; DE ARAÚJO, R. M. O.; DOS SANTOS, A. L. Sick leaves by mental disorders: Case study with public servants at an educational institution in Ceará, Brazil. Revista Brasileira de Medicina do Trabalho, v. 16, n. 1, p. 53-59, 2018. Disponível em: <http://www.rbmt.org.br/details/293/pt-BR>. doi: 10.5327/Z1679443520180167

BIAGGIO, A. M. B.; NATALÍCIO, L.; SPIELBERGER, C. D. Desenvolvimento da forma experimental em português do Inventário de Ansiedade Traço-Estado (IDATE) de Spielberger. Arquivos Brasileiros de Psicologia Aplicada, v. 29, n. 3, p. 31-44, 1977. Disponível em: <http://bibliotecadigital.fgv.br/ojs/index.php/abpa/article/view/17827>.

CARVALHO, E. A.; BERTOLINI, S. M. M. G.; MILANI, R. G.; MARTINS, M. C. Anxiety scores in university entering and graduating students from a higher education institution. Ciência, Cuidado e Saúde, v. 14, n. 3, p. 1290-1298, 2015. Disponível em: < http://periodicos.uem.br/ojs/index.php/CiencCuidSaude/article/view/23594>. doi: 10.4025/cienccuidsaude.v14i3.23594

DESOUKY, D.; ALLAM, H. Occupational stress, anxiety and depression among Egyptian teachers. Journal of Epidemiology and Global Health, v. 7, n. 3, p. 191198, 2017. Disponível em: < https://www.ncbi.nlm.nih.gov/pubmed/28756829>. doi: 10.1016/j.jegh.2017.06.002

FIKSDAL, A.; HANLIN, L.; KURAS, Y.; GIANFERANTE, D.; CHEN, X.; et al. Associations between symptoms of depression and anxiety and cortisol responses to and recovery from acute stress. Psychoneuroendocrinology, v. 102, p. 44-52, 2019. Disponível em: < https://www.ncbi.nlm.nih.gov/pubmed/30513499>. doi: 10.1016/j.psyneuen.2018.11.035

GERRETH, K.; CHLAPOWSKA, J.; LEWICKA-PANCZAK, K.; SNIATALA, R.; EKKERT, M.; et al. Self-Evaluation of Anxiety in Dental Students. BioMed Research International, v. 2019, p. 6436750, 2019. Disponível em: < https://www.hindawi.com/journals/bmri/2019/6436750/>. doi: 10.1155/2019/6436750

JOHNSON, S.; COOPER, C.; CARTWRIGHT, S.; DONALD, I.; TAYLOR, P.; et al. The experience of work-related stress across occupations. Journal of Managerial Psychology, v. 20, n. 2, p. 178-187, 2005. Disponível em: < https://www.lancaster.ac.uk/staff/taylorpj/papers/JMP2005.pdf>. doi: 10.1108/02683940510579803 
KULSOOM, B.; AFSAR, N. A. Stress, anxiety, and depression among medical students in a multiethnic setting. Neuropsychiatric disease and treatment, v. 11, n. 1 1, p. $\quad 1713-1722, \quad 2015 . \quad$ Disponível em: <http://www.ncbi.nlm.nih.gov/pubmed/26213470>. doi: 10.2147/NDT.S83577

LEAL, P. C.; GOES, T. C.; DA SILVA, L. C. F.; TEIXEIRA-SILVA, F. Trait vs. state anxiety in different threatening situations. Trends in Psychiatry and Psychotherapy, v. 39, n. 3, p. 147-157, 2017. Disponível em: < http://www.scielo.br/scielo.php?script=sci_arttext\&pid=S2237-60892017000300147> . doi: 10.1590/2237-6089-2016-0044

LEÃO, A. L. M.; BARBOSA-BRANCO, A.; RASSI NETO, E.; APARECIDA NEVES RIBEIRO, C.; TURCHI, M. D. Absenteísmo-doença no serviço público municipal de goiânia. Revista Brasileira de Epidemiologia, v. 18, n. 1, p. 262-277, 2015. Disponível em: < http://www.scielo.br/scielo.php?pid=S1415790X2015000100262\&script=sci_abstract\&tlng=pt>. doi: 10.1590/19805497201500010020

LOPES, S. V.; DA SILVA, M. C. Occupational stress and associated factors among civil servants of a federal university in the South of Brazil. Ciência e Saúde Coletiva, v. 23 , n. 11, p. 3869-3880, 2018. Disponível em: < http://www.scielo.br/scielo.php?script=sci_abstract\&pid=S1413-

$81232018001103869 \&$ Ing $=$ pt\&nrm=iso\&tIng=en>. doi:

$10.1590 / 1413-$

812320182311.28682015

LORICCHIO, T. M. B.; LEITE, J. R. Estresse, ansiedade, crenças de autoeficácia e o desempenho dos bacharéis em Direito. Avaliação Psicológica, v. 11, n. 1, p. 3747, $2012 . \quad$ Disponível em: <http://pepsic.bvsalud.org/scielo.php?script=sci_arttext\&pid=S167704712012000100005>.

MCCARTHY, J. M.; TROUGAKOS, J. P.; CHENG, B. H. Are anxious workers less productive workers? It depends on the quality of social exchange. Journal of Applied Psychology, v. 101, n. 2, p. 279-291, 2016. Disponível em: < https://www.ncbi.nlm.nih.gov/pubmed/26375962>. doi: 10.1037/apl0000044

MUSCHALLA, B. Different work capacity impairments in patients with different workanxieties. International Archives of Occupational and Environmental Health, v. 89, n. 4, p. 609-619, 2016. Disponível em: < https://link.springer.com/article/10.1007/s00420-015-1099-x>. doi: 10.1007/s00420015-1099-x

MUSCHALLA, B.; LINDEN, M. Different Workplace-Related Strains and Different Workplace-Related Anxieties in Different Professions. Journal of Occupational and Environmental Medicine, v. 55, n. 8, p. 978-982, 2013. Disponível em: $<$ http://content.wkhealth.com/linkback/openurl?sid=WKPTLP:landingpage \&an $=00043$ 764-201308000-00020>. doi: 10.1097/JOM.0b013e3182972f04

NUNES, A. V. L.; LINS, S. L. B. Servidores Públicos Federais: uma análise do prazer e sofrimento no trabalho. Revista Psicologia Organizações e Trabalho, v. 9, n. 1, p. 2009. 51-67, Disponível em: < 
https://periodicos.ufsc.br/index.php/rpot/article/view/11830>.

OLIVEIRA, L. A.; BALDAÇARA, L. R.; MAIA, M. Z. B. Afastamentos por transtornos mentais entre servidores públicos federais no Tocantins. Revista Brasileira de Saúde Ocupacional, v. 40, n. 132, p. 156-169, 2015. Disponível em: < http://www.scielo.br/scielo.php?script=sci_arttext\&pid=S0303-76572015000200156>. doi: 10.1590/0303-7657000092614

RAMAMOORTHY, S.; CIDLOWSKI, J. A. Corticosteroids. Mechanisms of Action in Health and Disease. Rheumatic Disease Clinics of North America, v. 42, n. 1, p. 15-31, 2016. Disponível em: < https://www.ncbi.nlm.nih.gov/pubmed/26611548>. doi: 10.1016/j.rdc.2015.08.002

REMES, O.; BRAYNE, C.; VAN DER LINDE, R.; LAFORTUNE, L. A systematic review of reviews on the prevalence of anxiety disorders in adult populations. Brain and Behavior, v. 6, n. 7, p. e00497, 2016. Disponível em: <http://doi.wiley.com/10.1002/brb3.497>. doi: 10.1002/brb3.497

REUMAN, L.; ABRAMOWITZ, J. S. Predictors of Accommodation Among Families Affected by Fear-Based Disorders. Child psychiatry and human development, v. 49, n. $1, \quad$ p. 53-62, $2018 . \quad$ Disponível em: <http://www.ncbi.nlm.nih.gov/pubmed/28409317>. doi: 10.1007/s10578-017-0728-x

ROBERTS, R. E.; DUONG, H. T. Is there an association between short sleep duration and adolescent anxiety disorders? Sleep Medicine, v. 30, p. 82-87, 2017. Disponível em: < https://www.ncbi.nlm.nih.gov/pubmed/28215269>. doi: 10.1016/j.sleep.2016.02.007

SARI, B. A.; KOSTER, E. H. W.; DERAKSHAN, N. The effects of active worrying on working memory capacity. Cognition and Emotion, v. 31, n. 5, p. 995-1003, 2017. Disponível em: <http://www.ncbi.nlm.nih.gov/pubmed/27064657>. doi: 10.1080/02699931.2016.1170668

SILVA, E. B. F.; TOMÉ, L. A. O.; COSTA, T. J. G. DA; SANTANA, M. C. C. P. Transtornos mentais e comportamentais: perfil dos afastamentos de servidores públicos estaduais em Alagoas, 2009. Epidemiologia e Serviços de Saúde, v. 21, n. 3, p. 505-514, 2012. Disponível em: < http://scielo.iec.gov.br/scielo.php?script=sci_arttext\&pid=S167949742012000300016>. doi: 10.5123/S1679-49742012000300016

SKAPINAKIS, P.; BELLOS, S.; KOUPIDIS, S.; GRAMMATIKOPOULOS, I.; THEODORAKIS P. N.; et al. Prevalence and sociodemographic associations of common mental disorders in a nationally representative sample of the general population of Greece. BMC Psychiatry, v. 13, n. 163, p. 1-14, 2013. Disponível em: <http://www.ncbi.nlm.nih.gov/pubmed/23734578>. doi: 10.1186/1471-244X-13-163

SPIELBERGER, C. D.; GORSUCH, R. L.; LUSHENE, R. E. Manual for the StateTrait Anxiety Inventory. 1. ed. Palo Alto, CA: Consulting Psychologists Press, 1970.

TREVISAN, R. L.; CRUZ, R. M.; BAASCH, D.; SOARES, D. P.; ROCHA, R. E. R.; et 
al. Prevalência de Transtornos do Humor e de Ansiedade em Servidores Públicos Afastados. Revista Psicologia em Pesquisa, v. 13, n. 2, p. 61-80, 2019. Disponível em: < https://periodicos.ufjf.br/index.php/psicologiaempesquisa/article/view/23845>. doi: 10.34019/1982-1247.2019.v13.23845

UMUZDAŞ, M. S.; TÖK, H.; UMUZDAŞ, S. An examination of the performance anxiety levels of undergraduate music teaching students in the instrument exams according to various variables (Case of Tokat province). International Journal of Higher Education, v. 8, n. 4, p. 221-230, 2019. Disponível em: < https://eric.ed.gov/?id=EJ1223641>. doi: 10.5430/ijhe.v8n4p221 